\title{
Trajectory Tracking Control of 3-PRP Parallel Robot Based on Fuzzy PI
}

\author{
Qiang SUN ${ }^{1}$, Zhen-zhong $\mathbf{Y U}^{2}$ and Qiang $\mathbf{L I}^{1}$ \\ ${ }^{1}$ Internet of Things Engineering School, Jiangnan University, \\ Jiangsu, Wuxi, 214122, China \\ ${ }^{2}$ Key Laboratory of Advanced Process Control for Light Industry of Ministry of Education, \\ Jiangnan University, Jiangsu, Wuxi, 214122, China
}

\begin{abstract}
Based on the characteristics of the trajectory planning of a parallel robot, the polynomial method was used to carry out the trajectory planning. Then, in view of the unstable factors in the control system of a parallel robot, a novel fuzzy PI controller was designed. Finally, the trajectory tracking performance of the 3PRP robot's joints were simulated with conventional PI and fuzzy PI controllers, respectively. The simulation results showed that the fuzzy PI controller could effectively enhance trajectory tracking precision.
\end{abstract}

Keywords: 3-PRP parallel robot, Trajectory planning, Fuzzy PI, Trajectory tracking control.

\section{Introduction}

As one of industrial robots, a parallel robot has a lot of advantages such as high precision, low cost, structural stability[1,2] and so on, which now has been applied in many fields. At present, the research on a parallel robot mainly focuses on control strategies[3] because a parallel robot is a strongly coupled system. And there are a number of unstable factors in the control system of a parallel robot. This will increase the parallel robot's trajectory tracking error.

In the early time, a nonlinear PI control strategy was applied to address this problem[4]. Due to the controller's limited ability to handle uncertainties, it may result in inaccurate and unstable control. Therefore, scholars have put forward fuzzy control strategy combined with PI control to reduce the trajectory tracking error of a parallel robot. Cao et al., based on the LabView software platform, designed a fuzzy PI controller, realizing the Delta parallel robot's high-precision control[5]; Javid et al. combined fuzzy PI and a terminal sliding mode controller to reach a good trajectory-tracking performance of the 3-PRR parallel manipulator[6]; Gao adopted PI and fuzzy PI to implement the joints' trajectory tracking of the Tripod parallel robot, respectively. And he found that the fuzzy PI controller had a better control performance[7].
As for the 3-PRP parallel robot, we employed the fuzzy PI control strategy to carry out the trajectory tracking of each joint, achieving high-precision control of the robot.

\section{Trajectory Planning}

\subsection{Description of the robot}

The structural diagram of the 3-PRP parallel robot is shown in Figure 1. And it is mostly made up of the static platform, the moving platform and three kinematic pairs connected with the static platform and the moving platform. Among the three motion pairs, the first moving pair and the second moving pair are symmetrical. And the joints of three kinematic pairs are all actuated by servo system.

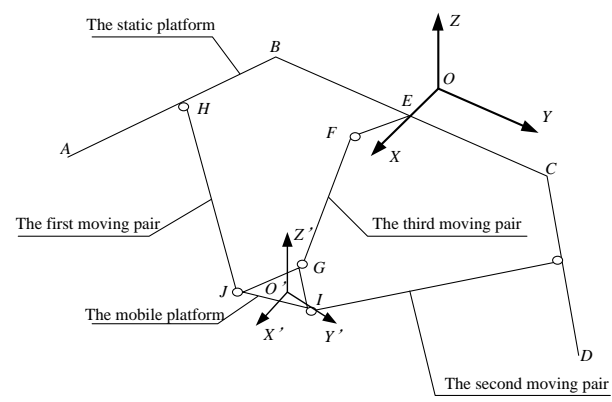

Fig. 1 The structural diagram of the 3-PRP parallel robot

\subsection{The polynomial method}

According to the different planning space, the trajectory planning of a parallel robot can be divided into two types: the operation space and the joint space. The trajectory planning in the operation space is directly to plan the position of the robot's moving platform and establish the mapping relationship between the position, the velocity, 
the accelerated velocity and time. The direct planning of the trajectory of the robot in the operation space can ensure that the moving platform of the robot moves along a specific path. In the case of avoidance of the obstacle and continuous trajectory tracking, this method is more effective than the trajectory planning in the joint space.

The purpose of the parallel robot's trajectory planning is that when the displacement and the maximum accelerated velocity are given, the path of the moving platform is optimal, that is to say, the motion time tends to be minimal. It requires that the velocity and the accelerated velocity of the moving platform are zero at the starting and the end time; The displacement curve of the robot's moving platform is continuous to one step and two step derivatives of the time, and the curves of the velocity and the accelerated velocity are smooth. At the same time, the accelerated velocity is as small as possible.

The moving platform of the parallel robot is commonly used for packing and sorting. Considering that there is a certain height or width obstacle in the process of the operation, the path is mostly composed of three straight lines, namely, $\overrightarrow{P_{1} P_{2}}\left(S_{1}\right), \overrightarrow{P_{2} P_{3}}\left(S_{2}\right), \overrightarrow{P_{3} P_{4}}\left(S_{3}\right)$, which constitute the gate-shaped trajectory. It is shown in Figure 2 .

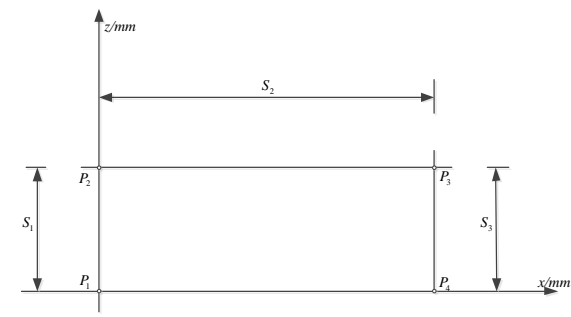

Fig. 2 Th path planning of the robot

Based on the principles of the given displacement and the maximum accelerated velocity, the polynomial method is adopted to carry out the trajectory planning. And the process of the trajectory planning can be described as follows:

(1) $S_{1}=S_{3}=25 \mathrm{~mm}<S_{2}=305 \mathrm{~mm}$;

(2) It assumes that the motion time of the moving platform in $S_{1}$ is $T_{1}$. Correspondingly, the motion time of $S_{2}$ and $S_{3}$ is $T_{2}$ and $T_{3}$. Also, the moments of $t=0, t=T_{1}, t=T_{2}$ are the initial time for $S_{1}, S_{2}$ and $S_{3}$, respectively;

(3) It is supposed that $\Gamma=\frac{t}{T}, H=\frac{s}{S}, V=\frac{v S}{s}, A=\frac{a T^{2}}{s}$, where, $t, a, s, v, T$ and $S$ denote the time, the accelerated velocity, the displacement, the velocity, the total time and the total displacement, respectively. Because the velocity and the accelerated velocity at the points of $P_{1}$ and $P_{4}$ are zero, it can be acquired:

$$
\left\{\begin{array}{l}
H=0, V=0, A=0(\Gamma=0) \\
H=1, V=0, A=0(\Gamma=1)
\end{array}\right.
$$

According to the Eq. (1), it can be derived with the 3-4-5 polynomial method:

$$
H=10 \Gamma^{3}-15 \Gamma^{4}+6 \Gamma^{5}
$$

It can be obtained by the derivation of Eq. (2):

$$
\left\{\begin{array}{l}
V=30 \Gamma^{2}-60 \Gamma^{3}+30 \Gamma^{4} \\
A=60 \Gamma-180 \Gamma^{2}+120 \Gamma^{3}
\end{array}\right.
$$

And the variable of $V$ and $A$ can be acquired:

$$
\left\{\begin{array}{l}
V=1.8750 \\
A=5.7735
\end{array}\right.
$$

Similarly, considering that the maximum accelerated velocity of the moving platform is $a_{\max }$, and $\Lambda=t / T$, it can be derived:

$$
\left\{\begin{array}{l}
s=\frac{a_{\max }}{5.7735} T^{2}\left(10 \Lambda^{3}-15 \Lambda^{4}+6 \Lambda^{5}\right) \\
v=\frac{a_{\max }}{5.7735} T\left(30 \Lambda^{2}-60 \Lambda^{3}+30 \Lambda^{4}\right) \\
a=\frac{a_{\max }}{5.7735}\left(60 \Lambda-180 \Lambda^{2}+120 \Lambda^{3}\right)
\end{array}\right.
$$

When $t$ is equal to $T$, it can be acquired:

$$
T=\sqrt{\frac{5.7335 S}{a_{\max }}}
$$

As for the 3-PRP parallel robot, the circumradius of the moving platform is $38 \mathrm{~mm}$; the length of three kinematic pairs is $500 \mathrm{~mm}$; the $B C$ of the static platform is $275 \mathrm{~mm}$. And $T=0.3 s, T_{1}=0.1 s, T_{2}=0.2 s, T_{3}=0.1 s$. This study assumes that the starting point and the end point of the robot's moving platform are set as $(305,0,-450)$ and $(-305$, $0,-450)$, and the velocity of the moving platform is $3 \mathrm{~m} / \mathrm{s}$. Based on the MATLAB software, the curves of the displacement, the velocity and the accelerated velocity in $S_{2}$ can be acquired as shown in Figure 3.

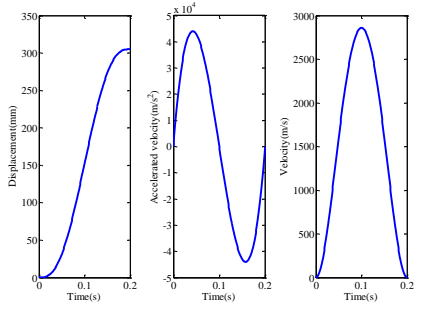

Fig. 3 Curves of displacement, acceleration, velocity in $S_{2}$

In a period of motion ( $T=0.3 s$ ), the displacements of $x, y$, $z$ axes and the path of the robot's moving platform are obtained, as shown in Figure 4 and Figure 5. 

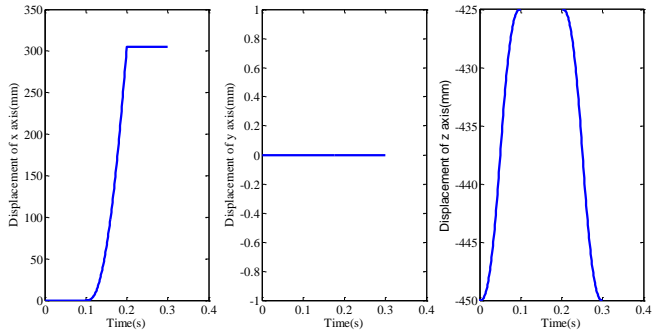

Fig. 4 Displacements of $\mathrm{x}, \mathrm{y}, \mathrm{z}$ axes

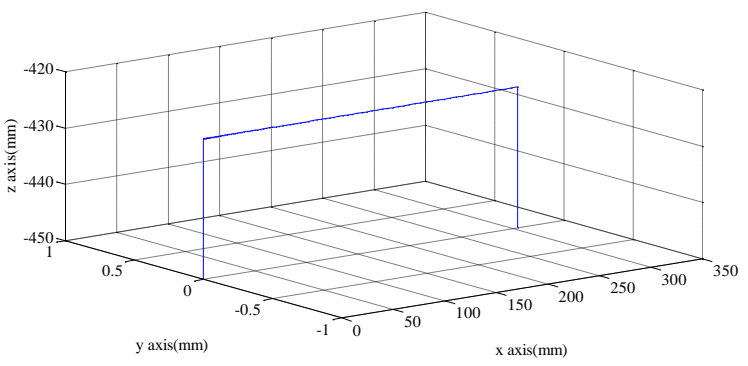

Fig. 5 Path of the robot's moving platform

And the curves of joints in the 3-PRP parallel robot were calculated by means of the inverse kinematic equations of the 3-PRP parallel robot. The results are shown in Figure 6.
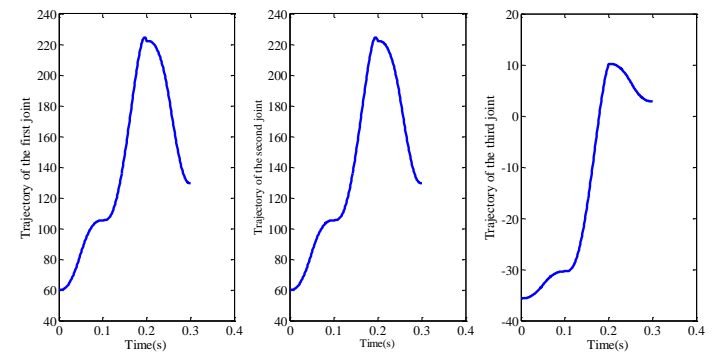

Fig. 6 Curves of joints of the 3-PRP robot

\section{Design of Fuzzy PI Controller}

PID control is capable of many advantages like good performance of stability, uncomplex structure, high reliability[8] and so on, which has been widely applied in industry. The 3-PRP parallel robot is a highly coupled system, and it is constantly disturbed by varying loads and nonlinear factors in the mechanism. If the traditional PID controller is used, the control parameters of the system can not be adjusted online according to the actual conditions, which will decline the performance of the control system and affect the normal work of the mechanism. Considering that the fuzzy control is adaptive to the nonlinear factors of the controlled object, we adopt fuzzy control strategy to realize online adjustment of the control system's parameters.

Because the speed loop in the servo system (the joints of the 3-PRP parallel robot are actuated by the servo system) has the function to restrain the disturbance of the load and the speed controller only contains modules of P and I, the speed controller can be defined as a fuzzy PI controller. The structure of the control system is shown in Figure 7.

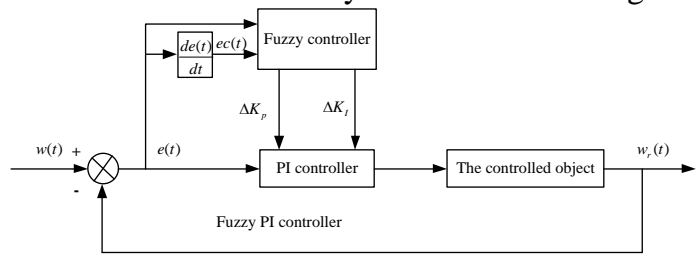

Fig. 7 The structure of the fuzzy PI controller

In the fuzzy controller, the input is the error value $e(t)$ and the error change rate $e c(t)$ between the actual angular velocity $w(t)$ and the given angular velocity $w_{r}(t)$, the output is the variable values $\Delta K_{P}$ and $\Delta K_{I}$ of the parameters $K_{P}$ and $K_{I}$ in the PI controller. Based on the $e(t)$ and the $e c(t)$, the fuzzy control can calculate the real-time values of $\Delta K_{P}$ and $\Delta K_{I}$ by the fuzzy reasoning, and transmit them to the PI controller in real time, realizing parameters' online adjustment of $K_{P}$ and $K_{I}$. The online adjustment of parameters in the PI controller is as follows:

$$
\left\{\begin{array}{l}
K_{P}=K_{P O}+\Delta K_{P} \\
K_{I}=K_{I O}+\Delta K_{I}
\end{array}\right.
$$

Where, $K_{P O}$ and $K_{I O}$ are the initial values in the PI controller.

In the fuzzy PI controller, $E$ and $E C$ are fuzzy variable of $e(t)$ and $e c(t) ; \Delta K_{P}$ and $\Delta K_{I}$ are fuzzy variable of $K_{P}$ and $K_{I}$. The designed domain of $E$ and $E C$ is $\{-6,-5,-4,-3,-2,-1$, $0,1,2,3,4,5,6\}$; the domain of $\Delta K_{P}$ and $\Delta K_{I}$ is $\{-60,-50$, $-40,-30,-20,-10,0,10,20,30,40,50,60\}$. And their fuzzy sets are $\{\mathrm{NB}, \mathrm{NM}, \mathrm{NS}, \mathrm{ZO}, \mathrm{PS}, \mathrm{PM}, \mathrm{PB}\}$, in which, $\mathrm{NB}$ is negative big, NM is negative middle, NS is negative small, $\mathrm{ZO}$ is zero, PS is positive small, PM is positive middle, PB is positive big. Both input and output membership functions are Gaussian function. Figure 8 is the membership function curve of each fuzzy variable.

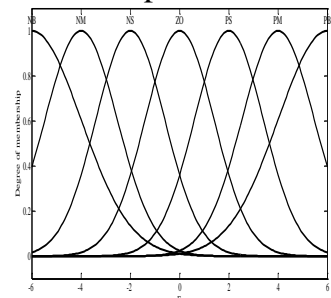

(a) Variable $E$

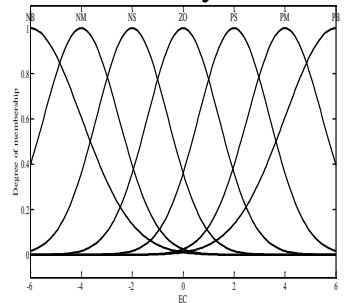

(b) Variable $E C$ 


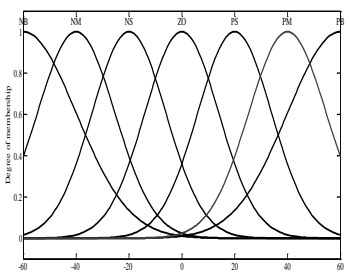

(c) Variable $\Delta K_{P}$

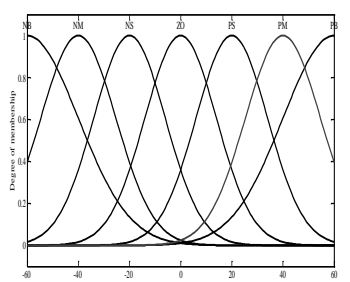

(d) Variable $\Delta K_{I}$
Fig. 8 Membership function of each fuzzy variable

In view of characteristics of the 3-PRP parallel robot, the design principle of the fuzzy rule is that when a larger $E$ is input, a large $\Delta K_{P}$ is selected and $\Delta K_{I}$ should be set as zero. This can guarantee the rapid response of the system; When the product of $E$ and $E C$ is greater than zero, it indicates that the deviation of the angular velocity is increasing. If the deviation is large, a large $\Delta K_{P}$ and a small $\Delta K_{I}$ are input to decrease the value of the deviation, improving the dynamic and steady-state performances of the system. If the deviation is small, a moderate $\Delta K_{P}$ and a large $\Delta K_{I}$ are input to ensure the improvement of the steady-state performance and the suppression of the oscillation of the system; When the product of $E$ and $E C$ is less than zero, it is indicated that the deviation is decreasing. A moderate $\Delta K_{P}$ and a small $\Delta K_{I}$ are input to achieve better steadystate and dynamic performance of the system. The designed fuzzy rules are shown in Table 1 and Table 2.

Table 1: Fuzzy rules of $\Delta K P$

\begin{tabular}{|l|l|l|l|l|l|l|l|}
\hline EC/E & NB & NM & NS & ZO & PS & PM & PB \\
\hline NB & PB & PB & PM & PM & PS & PS & ZO \\
\hline NM & PB & PB & PM & PM & PS & ZO & ZO \\
\hline NS & PM & PM & PM & PS & ZO & NS & NM \\
\hline ZO & PM & PS & PS & ZO & NS & NM & NM \\
\hline PS & PS & PS & ZO & NS & NS & NM & NM \\
\hline PM & ZO & ZO & NS & NM & NM & NM & NB \\
\hline PB & ZO & NS & NS & NM & NM & NB & NB \\
\hline
\end{tabular}

Table 2: Fuzzy rules of $\Delta K_{I}$

\begin{tabular}{|l|l|l|l|l|l|l|l|}
\hline EC/E & NB & NM & NS & ZO & PS & PM & PB \\
\hline NB & NB & NB & NB & NM & NM & ZO & ZO \\
\hline NM & NB & NB & PM & NM & NS & ZO & ZO \\
\hline NS & NM & NM & NS & NS & ZO & PS & PS \\
\hline ZO & NM & NS & NS & ZO & PS & PS & PM \\
\hline PS & NS & NS & ZO & PS & PS & PM & PM \\
\hline PM & ZO & ZO & PS & PM & PM & PB & PB \\
\hline PB & ZO & ZO & PS & PM & PB & PB & PB \\
\hline
\end{tabular}

According to the fuzzy rules, the Mamdani minimum method was used for fuzzy reasoning. And the defuzzification was implemented by gravity center method. The output reasoning surfaces of $\Delta K_{P}$ and $\Delta K_{I}$ are shown in Figure 9.

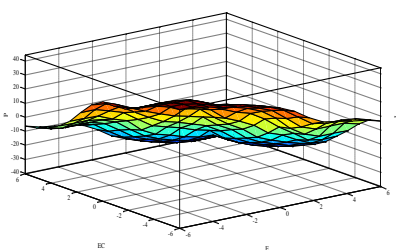

(a) $\Delta K_{P}$ fuzzy reasoning surface

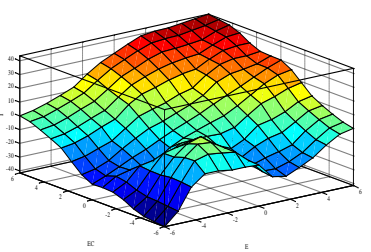

(b) $\Delta K_{I}$ Fuzzy reasoning surface
Fig. 9 Output surfaces of fuzzy reasoning

The designed fuzzy PI controller is shown in Figure 10.

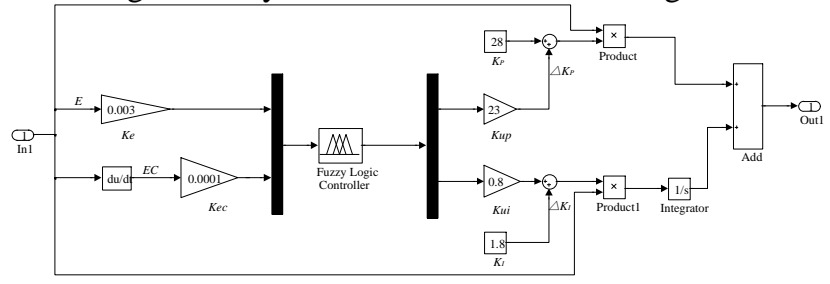

Fig. 10 The designed fuzzy PI controller in MATLAB

Where, the $K_{e}$ and the $K_{e c}$ are the quantification factors of $e(t)$ and $e c(t)$, respectively; correspondingly, the $K_{u p}$ and $K u i$ are the proportionality factors of $K_{P}$ and $K_{I}$.

\section{Simulation results and analysis}

The purpose of designing the fuzzy PI controller is to carry out the trajectory tracking of joints' angles of the 3-PRP parallel robot. Thus, a motion control system has been designed. The whole system consists of 3 subsystems, which are the position control module, the speed control module with fuzzy PI and the servo motor module. The input of the system is the angles of 3-PRP parallel robot's joints, the output is the angles of the servo motor. When the angles of joints are the inputs, the position controller will handle the value and transform it to the value of the angular velocity for the fuzzy PI controller, achieving the real-time adjustment of parameters of $K_{P}$ and $K_{I}$. For the realization of the equivalent control, the ratio of the angles of joints and the angles of servo motor is set as 1. Figure 11 is the simulation model of the control system in MATLAB/Simulink.

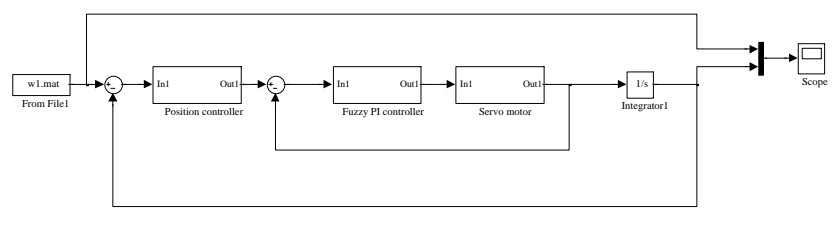

Fig. 11 Motion control system

Because the first motion pair and the second motion pair of the mechanism are symmetrical and their trajectories are 
equal, the first motion pair and the third motion pair are selected as the simulation objects. The data of Figure 6 is stored in file.mat and used as the simulation source. The configuration parameters are shown in Table 3.

Table 3: Simulation parameters

\begin{tabular}{|c|c|}
\hline The parameter name & The parameter value \\
\hline The position controller & $(28 s+1.8) / s$ \\
\hline The servo motor & $13.1345 s+5.909$ \\
\hline The initial value $K_{P}$ & 40 \\
\hline The initial value $K_{I}$ & 3 \\
\hline $\begin{array}{c}\text { The quantification } \\
\text { factor } K_{e}\end{array}$ & 0.003 \\
\hline $\begin{array}{c}\text { The quantification } \\
\text { factor } K_{e c}\end{array}$ & 0.0001 \\
\hline $\begin{array}{c}\text { The proportionality } \\
\text { factor } K_{u p}\end{array}$ & 23 \\
\hline $\begin{array}{c}\text { The proportionality } \\
\text { factor } K_{u i}\end{array}$ & 0.8 \\
\hline The simulation time $t$ & $0.3 \mathrm{~s}$ \\
\hline
\end{tabular}

The simulation results are shown in Figures 12 14.

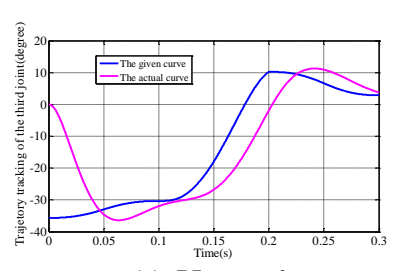

(a) PI control

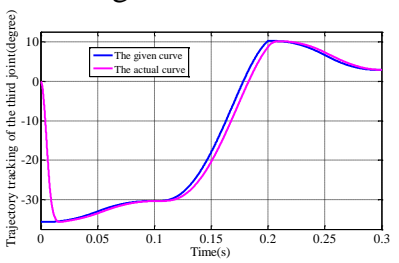

(b) Fuzzy PI control
Fig. 12 Tracking curves of the angles of the joint in the third moving pair

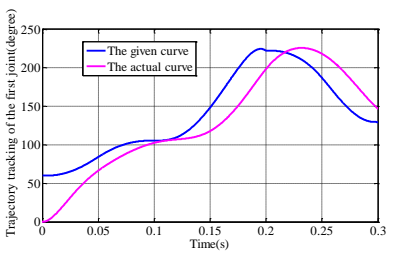

(a) PI control

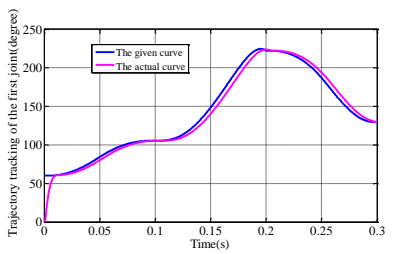

(b) Fuzzy PI control
Fig. 13 Tracking curves of the angles of the joint in the first moving pair

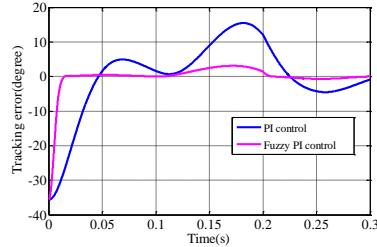

(a) Tracking error of the third joint

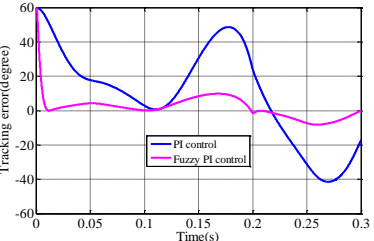

(b) Tracking error of the first joint
Fig. 14 Tracking error of each joint

In the Figures of 12 and 13, the blue line is the given angles of each joint, and the pink line is the simulation curve with PI and fuzzy PI control. The blue line and the pink line in the graph of 14 are the trajectory tracking error curves with PI and fuzzy PI control, respectively.

It can be seen from Figures 12 and 13, the PI control started to track the angles of each joint after the adjustment time of $0.06 \mathrm{~s}$. And the hysteresis times between the given curve and the actual curve in the third and the first joints are $40 \mathrm{~ms}$ and $37 \mathrm{~ms}$, which are $13.3 \%$ and $12.3 \%$ of the whole simulation period (which is equal to the motion period), respectively. The outcomes show that the PI control is poor in the real-time and dynamic performances. The fuzzy PI controller begins to track the angles of each joint after the adjustment time of $0.015 \mathrm{~s}$, which is shorter than the PI controller. This indicates that the fuzzy PI controller is better than the PI controller in the dynamic response. In addition, the hysteresis times between the given curve and the actual curve in the third and the first joints are $10 \mathrm{~ms}$ and $5 \mathrm{~ms}$, which are 3.3\% and $1.6 \%$ of the whole motion period, respectively, suggesting the fuzzy PI controller can improve the hysteretic performance of the control system.

According to the Figure 14, the mean tracking errors of the angles in the third and the first joints using the PI controller are -1.4139 degrees and +9.9592 degrees, respectively. Using the fuzzy PI controller, the mean tracking errors of the angles in the third and the first joints are -0.8503 degrees and +2.2869 degrees, respectively. The tracking precision of the angles of the third and the first angles are separately enhanced by $39.9 \%$ and $77 \%$. Because of the unstable factors in the parallel robot and the poor adaption of the PI controller to these factors, there exits huge errors of the trajectory tracking. While, the fuzzy PI controller can calculate the parameters online in the control system and finally enhance the precision of the trajectory tracking of each joint.

\section{Conclusions}

In view of the characteristics of the parallel robot, the 3-45 polynomial method was adopted to carry out the trajectory planning of the 3-PRP parallel robot in the operation space. Then, in order to improve the adaption to the unstable factors in the control system of the robot, a fuzzy PI controller has been designed. Finally, the trajectory tracking performance of each joint were simulated by PI and fuzzy PI controllers. According to the simulation results, the fuzzy PI controller was more adaptive to the unstable factors than the PI controller and enhanced the precision of the trajectory tracking.

\section{Acknowledgments}

This work is financially supported by the natural science foundation of Jiangsu Province of China. 


\section{References}

[1] Stewart D, "A platform with six degrees of freedom", Proceedings of the Institution of Mechanical Engineers, 1965, pp. 371-386.

[2] H. Maccallion, D. T. Pham, "The analysis of a six degrees of freedom work station for mechanized assembly" in Proc. 5sth World Congress on Theory of Machines and Mechanisms, 1979, pp. 611-616.

[3] Shuang CONG, Yao-xin ZHANG, "Control strategies of parallel manipulator systems" in 2005 Chinese Control and Decision Conference, 2005, pp. 1973-1977.

[4] Nabat. V, Rodruguez MDLO, Company. O, Krut, S, Pierrot. F, "Par4: Very high speed parallel robot for pick-and-place" in IEEE International Conference on Intelligent Robots and Systems, 2005, pp. 554-558.

[5] Qin-jie CAO, Zhao-rui LIANG, Wu-tong JIA, "Delta Robot Motion Control System Based on Fuzzy PI", Measurement \& Control Technology, 34,7, 2015, pp. 90-93.

[6] Javid. G, Akbarzadeh-T. M. R, Akbarzadeh. A, Nabavi SN, "Trajectory tracking of 3-PRR parallerl manipulator with PI Adaptive fuzzy terminal sliding mode controller" in IEEE International Econference on Computer and Knowledge Engineering, 2013, pp. 156-161.

[7] Jun-dong GAO, "Kinematics analysis and Motion control research of Tripod Parallel Robot", M.S. thesis, College of Mechanical Engineering, Chongqing University, Chongqing, China, 2016.

[8] Qing-jue HAN, Li-jun LI, Hui CHEN, "Slip control of deepsea tracked miner during walking", International Journal of Computer Science Issues, 14, 5, 2017, pp. 1-5.

Qiang SUN has received the B.Sc. in 2015 in the College of Internet of Things Engineering from Jiangnan University, China. Now he is a master at school in Jiangnan University of College of Internet of Things Engineering. His major research focuses on the motion control of the parallel robot. Mr. SUN is a membership of HIIRI.

Zhen-zhong YU has received PhD in 2010 in Harbin Institute of Technology. Now he is a vice professor in the College of Internet of Things of Jiangnan University. 